\title{
New and revised parameters for several southern OB binaries ${ }^{\star}$
}

\author{
Pavel Mayer ${ }^{1}$, Horst Drechsel ${ }^{2}$, and Andreas Irrgang ${ }^{2}$ \\ 1 Astronomical Institute of the Charles University, Faculty of Mathematics and Physics, V Holešovičkách 2, \\ Praha 8180 00, Czech Republic \\ e-mail: mayer@cesnet.cz \\ 2 Dr. Karl-Remeis-Observatory \& ECAP, Astronomical Institute, Friedrich-Alexander University Erlangen-Nuremberg, \\ Sternwartstr. 7, 96094 Bamberg, Germany
}

Received 17 January 2014 / Accepted 6 March 2014

\section{ABSTRACT}

Using ESO FEROS archive spectra of several southern OB-type binaries, we derived periods for three SB2 spectroscopic binaries, HD 97166, HD 115455, and HD 123590, and two SB1 systems, HD 130298 and HD 163892. It was also possible to use new FEROS spectra to improve the parameters of the known binaries, KX Vel and HD 167263. For KX Vel, we determined a dynamic mass of the primary of $16.8 M_{\odot}$, while the evolutionary model suggests a higher value of $20.2 M_{\odot}$. We derived an improved period for HD 167263 , and in its spectra, we recognized contributions of both of its interferometric components.

Key words. stars: early-type - binaries: spectroscopic

\section{Introduction}

Massive stars of spectral types $\mathrm{O}$ and early $\mathrm{B}$ deserve special attention because these extremely rare objects are the main drivers of the chemical and dynamical evolution of galaxies and play an important role as cosmological probes. They are crucial in the comparison to recent refinements of the theory of stellar structure and evolution of massive stars with respect to mass loss, core overshooting, rotation, and metallicity. The still persisting discrepancy between expected and observed masses of very massive stars also needs to be resolved. In particular, binaries offer the opportunity to provide precise absolute stellar quantities (masses, radii, and luminosities) for challenging theoretical predictions. Listings of O-type stars such as those by Mason et al. (1998) and Maíz-Apellániz et al. (2004) contain many early-type objects, for which their status as single or double stars - either SB1 or SB2 - is still uncertain. Recently, Chini et al. (2012) presented an extensive spectroscopic survey of OB stars regarding their binarity, which was based on several thousand spectra obtained with the ESO FEROS and Bochum University BESO echelle spectrographs over the past years.

The FEROS ${ }^{1}$ archive of ESO is a rich source of spectroscopic data. It contains a wealth of O-type star spectra, most of which can be directly used in pipeline-reduced form. In the course of ourlong-term project to analyze early-type binaries and multiple systems, we mainly used FEROS archive data to

* Based on data products from observations made with ESO Telescopes at the La Silla Paranal Observatory under program ID: 074.D-0300(A), 075.D-0061(A), 075.D-0103(A), 077.B-0348(A), 077.D-0321(A), 077.D-0635(A), 079.D-0564(A)， 079.D-0564(B), 081.D-2008(A), 081.D-2008(B), 083.D-0589(A)， 086.D-0997(A), 087.D-0946(A), 089.D-0189(A), and 089.D-0975(A).

1 FEROS is an echelle fiber spectrograph used with the $2.2 \mathrm{~m}$ telescope MPIA/ESO at La Silla and supplies spectra with the resolving power of 48000 in the region from 3575 to $9215 \AA$. identify hitherto unknown binaries among $\mathrm{O}$ stars and to obtain their fundamental parameters. An example of an already published study is the paper on HD 165246 (Mayer et al. 2013).

Here, we present additional new results based on the study of the FEROS spectra of several southern O-type stars. Three of the stars - HD 97166, HD 115455, and HD 123590 - were found to be double-lined spectroscopic binaries; HD 130298 and HD 163892 could be identified as single-lined binaries (Sect. 2). We measured the radial velocities (RVs) using Gaussian fits. In a recent paper by Sota et al. (2014), approximate periods for four of these five binaries were published. Our values were found independently of this paper. In Sect. 3, we present a revised study of the two OB binaries KX Vel and HD 167263 and derive improved parameters based on an analysis of new FEROS spectra.

\section{New radial velocity curves and orbital periods for five OB binaries}

\section{1. $H D 97166$}

According to Walborn (1973), HD 97166 is an O7.5 III((f)) star; Sota et al. (2011) give a slightly different classification of this binary as O8 IV (in their Table 2). With a $V$ magnitude of 7.89, it is the brightest member of the open cluster NGC 3572 in the Carina region. Chini et al. (2012) have noted the SB2 nature of this object. The sample of the FEROS spectra that we analyzed is listed in Table 1 with the measured RVs. In most spectra, the primary and secondary lines are strongly blended. Fortunately, the assignment of lines to both components and the measurement of their RVs is facilitated by considerably different temperatures of both components: for example, lines of Si III originate only in the cooler component, while the contribution of the hotter component is strong in He II lines, as seen in Fig. 4. Therefore, we first measured the narrow Si III lines 4553 and $4568 \AA$, for which 
Table 1. Spectra and RVs of HD 97166.

\begin{tabular}{lrrr}
\hline \hline $\begin{array}{l}\text { HJD } \\
-2400000\end{array}$ & $\begin{array}{r}\text { RV Pri } \\
\mathrm{km} \mathrm{s}^{-1}\end{array}$ & $\begin{array}{r}\text { RV Sec } \\
\mathrm{km} \mathrm{s}^{-1}\end{array}$ & Phase \\
\hline 42890.5900 & $-4.0^{1}$ & & 0.6236 \\
54211.7307 & 68.0 & -98.6 & 0.8404 \\
54599.5973 & -28.0 & 17.0 & 0.5587 \\
54600.6240 & -24.5 & 13.0 & 0.5787 \\
54601.5756 & -18.0 & 9.8 & 0.5929 \\
54625.6280 & 74.0 & -106.3 & 0.0096 \\
54626.6215 & 48.0 & -76.2 & 0.0268 \\
54953.6701 & 13.0 & -17.6 & 0.6916 \\
54956.6974 & 31.0 & -36.4 & 0.7440 \\
55605.8678 & 93.0 & -130.4 & 0.9884 \\
55643.7316 & -10.6 & -6.5 & 0.6442 \\
56067.6708 & 97.0 & -136.4 & 0.9873 \\
56098.5979 & -32.0 & 29.8 & 0.5230 \\
\hline
\end{tabular}

Notes. RV published by Conti et al. (1977); only the primary RV is given.

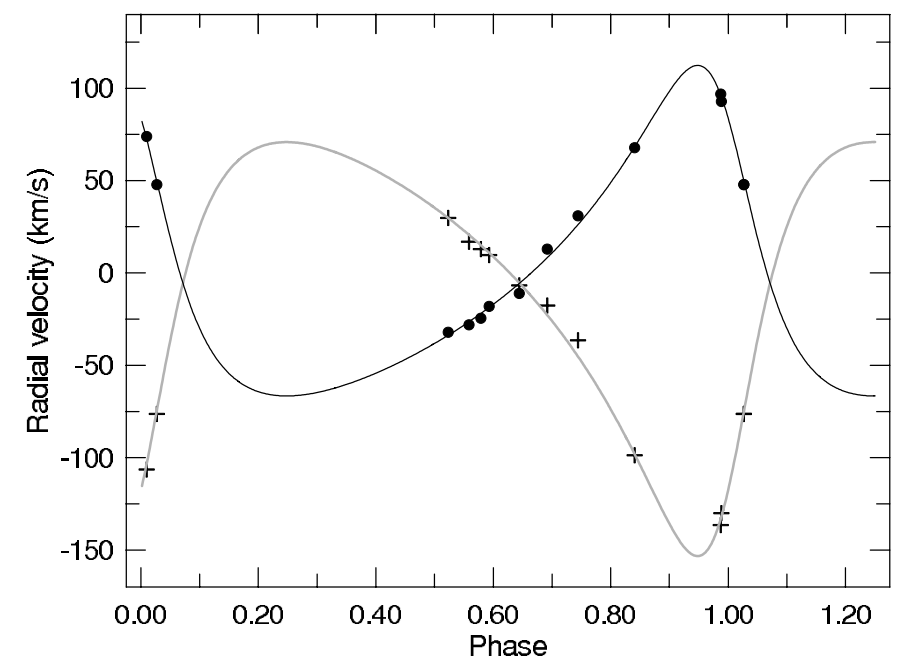

Fig. 1. Radial velocity curve of HD 97166 (FEROS spectra); full circles - primary RVs, crosses - secondary RVs.

an accuracy of about $1 \mathrm{~km} \mathrm{~s}^{-1}$ can be achieved. In a second step, the deconvolution of the line blends and the measurement of the line positions of the hotter component were carried out by keeping the RVs of the cooler component at their previously determined values.

We found only one published RV of this star. It was measured by Conti et al. (1977) and is contained in our Table 1. The error in phase for this old RV is \pm 0.06 , which cannot explain the deviation of this measurement from our RV curve. The use of such an old RV could potentially improve the accuracy of the period due to its large time separation, but it would be questionable because of the severe blending of the primary and secondary lines. It was, therefore, not included in our data set.

The values of $m_{i} \sin ^{3} i$ for the binary components of HD 97166 are large. The mass of the primary component can be estimated at about $24 M_{\odot}$ using a calibration of $\mathrm{O}$ star parameters by Martins et al. (2005). Hence, an inclination of $\approx 70^{\circ}$ can be estimated. Nevertheless, owing to the large separation of the components, no eclipses or ellipsoidal variations are to be expected or have so far been mentioned in the literature.
Table 2. Spectra and RVs of HD 115455.

\begin{tabular}{lrrl}
\hline \hline $\begin{array}{l}\text { HJD } \\
-2400000\end{array}$ & $\begin{array}{r}\text { RV Pri } \\
\mathrm{km} \mathrm{s}^{-1}\end{array}$ & $\begin{array}{r}\text { RV Sec } \\
\mathrm{km} \mathrm{s}^{-1}\end{array}$ & Phase \\
\hline 53373.8182 & 3.5 & -51 & 0.6017 \\
53965.4890 & -50.7 & 72 & 0.8265 \\
53966.4905 & -69.8 & 90 & 0.8929 \\
53967.4806 & -75.0 & 98 & 0.9585 \\
53968.4802 & -59.0 & 70 & 0.0248 \\
53969.4817 & -31.4 & 21 & 0.0912 \\
53970.4942 & -6.7 & -49 & 0.1583 \\
54191.7093 & -53.5 & 62 & 0.8237 \\
54209.7216 & -59.6 & 82 & 0.0179 \\
54209.7350 & -61.2 & 72 & 0.0187 \\
54211.7461 & -9.6 & -43 & 0.1521 \\
54212.7669 & 7.9 & -70 & 0.2197 \\
54244.6680 & 23.0 & -103 & 0.3346 \\
54247.6990 & 14.7 & -85 & 0.5356 \\
54267.5071 & -60.5 & 77 & 0.8488 \\
\hline
\end{tabular}

To determine spectral types, we used the $\log W^{\prime}$ criterion by Conti \& Alschuler (1971), which is based on the ratio $W^{\prime}$ of equivalent widths for the lines He I $4471 \AA$ and He II $4541 \AA$. The values of $\log W^{\prime}$ are 0.05 for the primary and $\approx 0.4$ for the secondary components, respectively. The former value agrees with the original classification of 07.5 by Walborn (1972), and the latter value suggests a type 09 for the secondary component, which is probably of luminosity class V. The luminosity ratio $L_{2} / L_{1}$ can be estimated from the strength of the He I line $5876 \AA$, since this line is nearly independent of temperature and $\log g$ in the possible parameter range for this system. Accordingly, the ratio $L_{2} / L_{1}$ is 0.45 , which corresponds to a magnitude difference of 0.85 . Therefore, a compromise classification of the primary as a class O7.5 IV object is suggested. Adopting the calibration by Martins et al. (2005), the absolute magnitudes of the primary and secondary components are $\approx-5.0$ and -3.9 , respectively. With an integral absolute magnitude of -5.34 , an observed $V$ brightness of 7.87 and an absorption of $A_{V}=1.28$, we obtain an absolute visual magnitude of $M_{V}=-5$ m. This implies a distance modulus of 11.93 for HD 97166, which is close to the value of 12 m 22 found by Moffat \& Vogt (1975) for the cluster NGC 3572. Elements of the orbit are contained in Table 6.

\section{2. $H D 115455$}

The binary HD 115455 is another O7.5 III((f)) star (Walborn 1973). It is a member of the open cluster Stock 16 with $V=7.95$. The RVs of its primary component were published by Crampton (1972, 5 RVs), who noted the RV variability, and Stickland \& Lloyd (2001, 1 RV; SL). It was possible to include these old velocities in our calculation of the period, resulting in a considerable improvement of its accuracy.

The new primary velocities were obtained from the He II lines 4541 and $5411 \AA$ A. Since the cooler secondary does not produce a measurable contribution to these lines, the He I lines 4922 and $5876 \AA$ were used to determine the RVs of this binary component. All RVs are listed in Table 2, and the RV curve is shown in Fig. 2. Two deviating RVs by Crampton (1972) might be affected by the vicinity of the conjunction. Orbital elements are presented in Table 6. Examples of two 
P. Mayer et al.: Several southern OB binaries

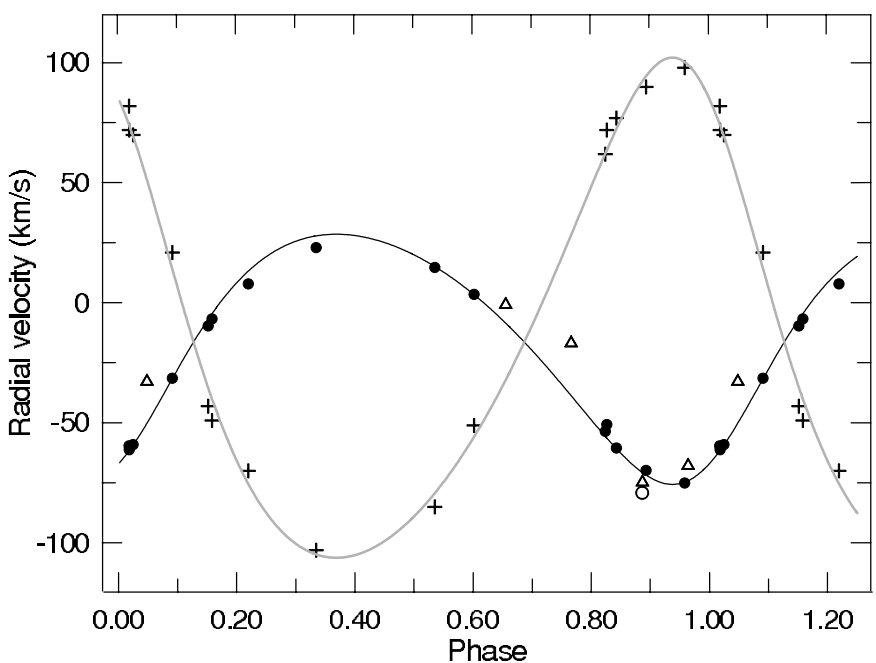

Fig. 2. Radial velocity curve of HD 115455. Primary RVs: full circles FEROS, triangles - Crampton (1972), open circle - SL. Secondary RVs: crosses - FEROS.

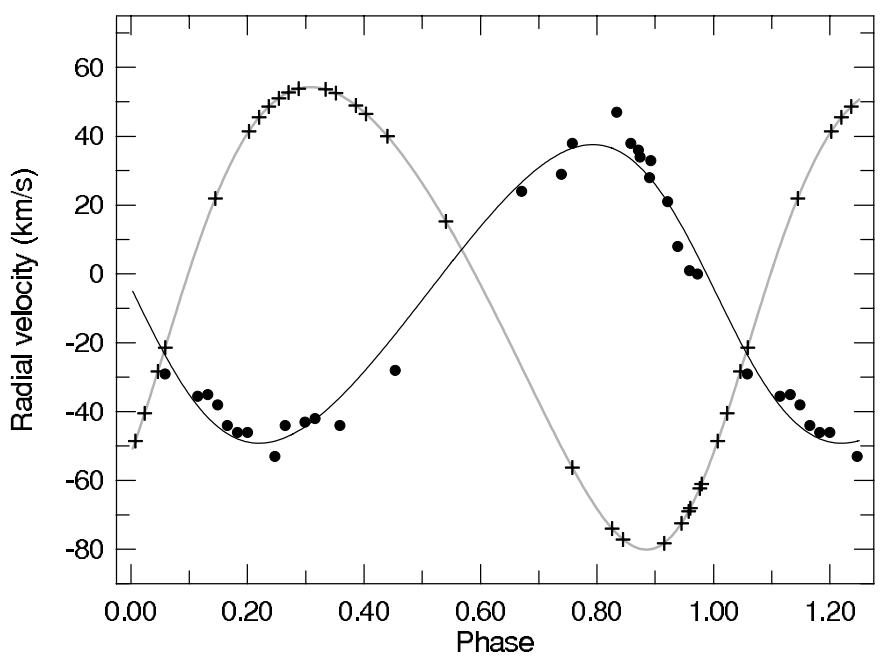

Fig. 3. Radial velocity curve of HD 123590. Primary RVs: full circles. Secondary RVs: crosses.

profiles of the He I $4922 \AA$ line close to the quadrature phases are shown in Fig. 4.

As in the subsection 2.1, $L_{2} / L_{1}$ was estimated from the He I line $5876 \AA$ A. Except for the He I lines, the primary and secondary components can also be discerned in $\mathrm{Mg}$ II and Si III lines, while O II lines can only be attributed to the secondary component. Although the intensity ratios of various lines give slightly different results, $L_{2} / L_{1}$ equals about 0.10 . The secondary spectral type can therefore be estimated as B2 V.

The distance to the cluster Stock 16 was given as $1900 \pm$ 100 pc by Vázquez et al. (2005). However, given the expected total magnitude of the binary of $M_{V}=-5.5$ and $V_{0}=6.4$, the distance would be $2400 \mathrm{pc}$.

\section{3. $H D 123590$}

For this $\operatorname{star}(V=7.62)$, no classification based on a slitspectrogram is available. It is an SB2 system with wide and narrow line components. The wide component has the smaller $\mathrm{RV}$ amplitude, so it belongs to the primary. We estimate the spectral type of both components as O7. However, the narrow
Table 3. Spectra and RVs of HD 123590.

\begin{tabular}{lrrl}
\hline \hline HJD & RV Pri & $\begin{array}{r}\text { RV Sec } \\
-2400000\end{array}$ & Phase \\
\hline 53965.4771 & -35 & 42.2 & 0.2015 \\
53966.5065 & -35 & 44.5 & 0.2190 \\
53967.4946 & -38 & 48.9 & 0.2357 \\
53968.4942 & -44 & 50.8 & 0.2527 \\
53969.4958 & -46 & 53 & 0.2697 \\
53970.5140 & -46 & 55.3 & 0.2870 \\
54191.7552 & 1 & -29.0 & 0.0454 \\
54209.7485 & -44 & 52.7 & 0.3510 \\
54211.7601 & -43 & 48.8 & 0.3825 \\
54212.7774 & -42 & 45.1 & 0.4025 \\
54244.6799 & 38 & -71.9 & 0.9444 \\
54246.6991 & 33 & -62.6 & 0.9787 \\
54267.5692 & -53 & 54.2 & 0.3332 \\
54292.4910 & 24 & -57.1 & 0.7566 \\
54296.5114 & 29 & -73.2 & 0.8249 \\
54297.6247 & 38 & -77.1 & 0.8438 \\
54338.5755 & -28 & 15.3 & 0.5395 \\
54480.8590 & 36 & -68.6 & 0.9565 \\
54539.8975 & 34 & -67.2 & 0.9594 \\
54542.6808 & 21 & -49.7 & 0.0067 \\
54545.6954 & 0 & -22.1 & 0.0579 \\
54550.7541 & -29 & 21.3 & 0.1438 \\
54599.7173 & 28 & -62.8 & 0.9756 \\
54955.6960 & 8 & -40.8 & 0.0228 \\
56067.7963 & 47 & -77.8 & 0.9145 \\
56098.6775 & 44 & 40.4 & 0.4391 \\
\hline & & &
\end{tabular}

component is also present in the He II line $6683 \AA$, so its luminosity might be higher than that of the wide component. This is also supported by the width of the He II line $5411 \AA$, as seen in Fig. 4: it is known that the lower $g$ lines are intrinsically wider (Lanz \& Hubeny 2007). Due to the strong blending of the broad lines, we did not attempt to measure RVs of He II lines.

The measured RVs are listed in Table 3, and the elements of the orbit are given in Table 6. Rotational velocities of both components differ considerably, as seen in Fig. 4.

\subsection{HD 130298}

This is the earliest binary in our sample with type O6III(f) (Garrison et al. 1977) and $V=9.23$. Four RVs were published and their variability was noted by Feast et al. (1963). Although it is listed as an emission-line star in SIMBAD, no emission contributions are apparent in the FEROS spectra in the $\mathrm{H} \alpha$ or He II $4686 \AA$ profiles. Sota et al. (2014) gave an approximate period of 14.63 . We refined this value to 14.6302 and calculated the other elements of the orbit (Table 6). The residuals of the FEROS RVs are very small, as documented by Fig. 5. The new period is also compatible with the RVs measured by Feast et al. (1963), although the scatter of these measurements is large $\left(\mathrm{rms}=20 \mathrm{~km} \mathrm{~s}^{-1}\right)$. Despite the claim by Chini et al. (2012) that the object type could be SB2, no traces of the secondary component are evident in the He I, Si III, or O II lines.

\subsection{HD 163892}

This O9 IV star (Walborn 1973) with brightness $V=7.44$ is a member of the association Sgr OB1 (Humphreys 1978). Several 

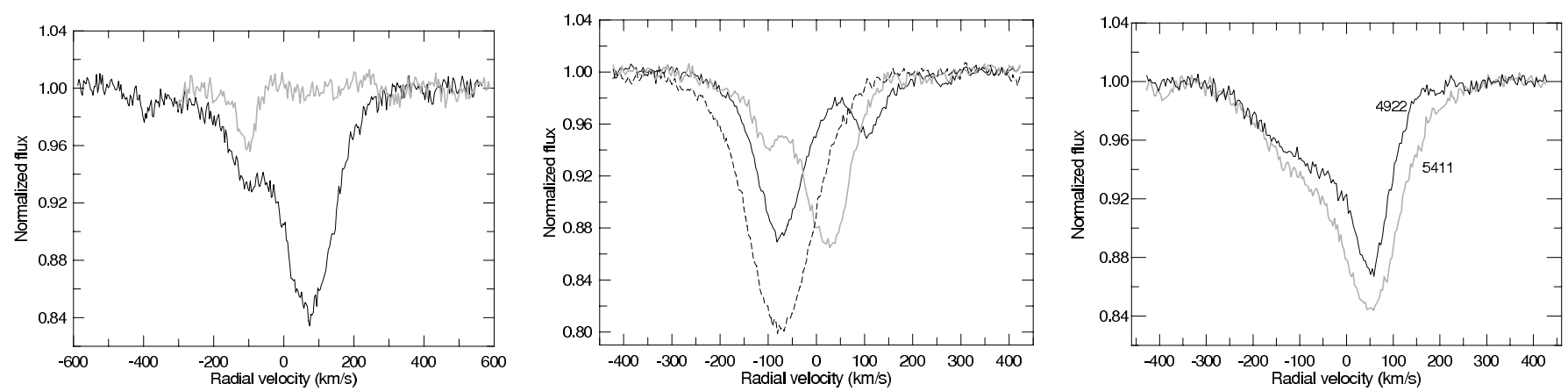

Fig. 4. Examples of line profiles of three stars. Left: HD 97166; Si III $4553 \AA$ (gray) and He II $5411 \AA$ (black) in the spectrum of JD 2454625. Middle: HD 115455; lines He I $4922 \AA$ (black) and He II $5411 \AA$ (dashed) at JD 2453967 and He I $4922 \AA$ at 2454244 (gray). Right: HD 123590; He I $4922 \AA$ and He II $5411 \AA$ at JD 2453970.

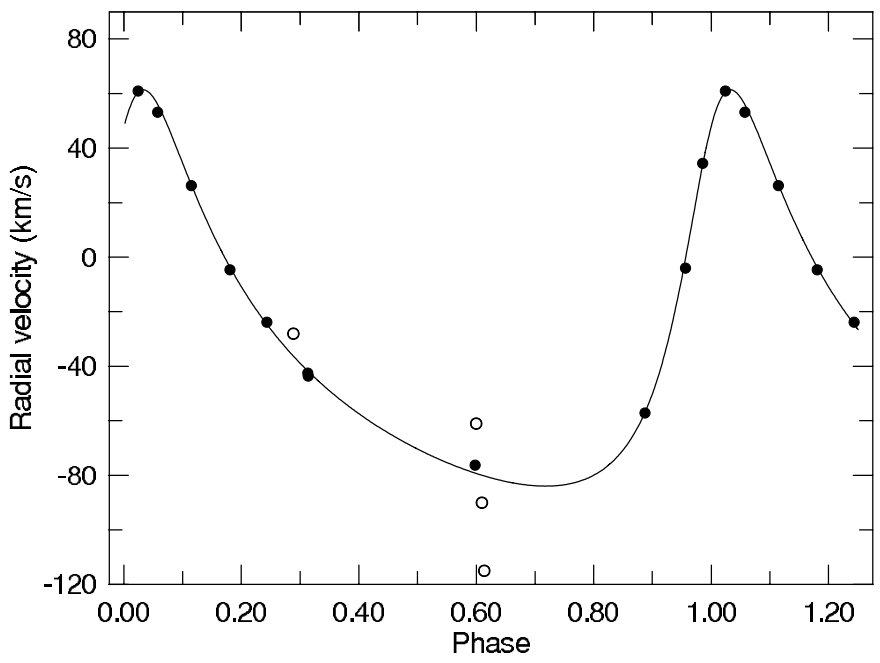

Fig. 5. Radial velocity curve of HD 130298. Full circles - RVs from FEROS spectra, open circles - RVs by Feast et al. (1963).

RVs were already published: Feast et al. (1963), who noted their variability, Conti et al. (1977), and SL. These RVs are included in Table 5. Although the RV curve shown in Fig. 7 appears realistic, it should be noted that the average RV of the association members is about $-10 \mathrm{~km} \mathrm{~s}^{-1}$, but the binary $V \gamma$ differs by $\approx 14 \mathrm{~km} \mathrm{~s}^{-1}$. The solution of the orbit is given in Table 6 . We tried to find any indications for the presence of the secondary component but without success. The secondary He I lines have to be at least 20 times fainter than the primary ones.

\section{New orbital elements for two binaries}

\section{1. $K X \mathrm{Vel}$}

The number of eclipsing binaries with an evolved early-type component is rather small. For such systems, direct and accurate determinations of their masses are important but have rarely been reported. The binary KX Vel (HR 3527, HD 75821, HIP 43413, $V=5.10$ ) is of type B0 III, according to Morgan et al. (1955), and belongs to this group of OB-type systems. Balona \& Laing (1986) discovered that this star is an eclipsing variable; hence, a determination of the component masses is in principle possible. Electronic RVs of the star were published by Mayer et al. (1997, MLD) and by SL. However, the secondary velocities were determined at only three phases; therefore, the secondary semi-amplitude $K_{2}$ was rather uncertain. Now several more recent spectra obtained with FEROS are available.
Table 4. Spectra and RVs of HD 130298.

\begin{tabular}{lrcc}
\hline \hline $\begin{array}{l}\text { HJD } \\
-2400000\end{array}$ & $\begin{array}{r}\text { RV Pri } \\
\mathrm{km} \mathrm{s}^{-1}\end{array}$ & Phase & \\
\hline 34578.280 & -115 & 0.6132 & $\mathrm{~F}$ \\
34851.501 & -28 & 0.2882 & $\mathrm{~F}$ \\
34973.241 & -90 & 0.6095 & $\mathrm{~F}$ \\
45236.444 & -61 & 0.5999 & $\mathrm{~F}$ \\
54211.7824 & -76.2 & 0.5977 & \\
54246.7136 & 34.5 & 0.9853 & \\
54247.7661 & 53.2 & 0.0573 & \\
54599.7295 & 26.3 & 0.1146 & \\
54600.6947 & -4.6 & 0.1806 & \\
54601.6135 & -23.8 & 0.2434 & \\
54625.6620 & -57.1 & 0.8871 & \\
54626.6763 & -4.0 & 0.9565 & \\
54627.6641 & 61.0 & 0.0240 & \\
54953.7548 & -42.5 & 0.3128 & \\
54953.7695 & -43.6 & 0.3139 & \\
\hline
\end{tabular}

Notes. Source: F - Feast et al. (1963). The remaining spectra are FEROS spectra.

Table 5. Spectra and RVs of HD 163892.

\begin{tabular}{lrcc}
\hline \hline $\begin{array}{l}\text { HJD } \\
-2400000\end{array}$ & $\begin{array}{r}\text { RV Pri } \\
\mathrm{km} \mathrm{s}^{-1}\end{array}$ & Phase & Source \\
\hline 34848.5900 & -15 & 0.0085 & $\mathrm{~F}$ \\
34910.3790 & $13:$ & 0.8950 & $\mathrm{~F}$ \\
35565.5690 & 18 & 0.5220 & $\mathrm{~F}$ \\
40044.8300 & -35.4 & 0.2448 & $\mathrm{C}$ \\
46680.8790 & -27.4 & 0.2551 & $\mathrm{SL}$ \\
53546.8814 & 37.3 & 0.6161 & \\
54600.8870 & -36.1 & 0.1470 & \\
54626.8489 & 7.5 & 0.4607 & \\
54953.9244 & -37.4 & 0.2079 & \\
54954.8706 & -19.6 & 0.3287 & \\
54955.7251 & 3.4 & 0.4377 & \\
54956.7811 & 30.8 & 0.5725 & \\
54976.8538 & -38.1 & 0.1345 & \\
56059.8150 & -13.6 & 0.3612 & \\
56067.9061 & -5.5 & 0.3940 & \\
56097.8446 & -40.9 & 0.2123 & \\
\hline
\end{tabular}

Notes. Source: F - Feast et al. (1963), C - Conti et al. (1977), SL Stickland \& Lloyd (2001). The remaining spectra are FEROS spectra. 
P. Mayer et al.: Several southern OB binaries
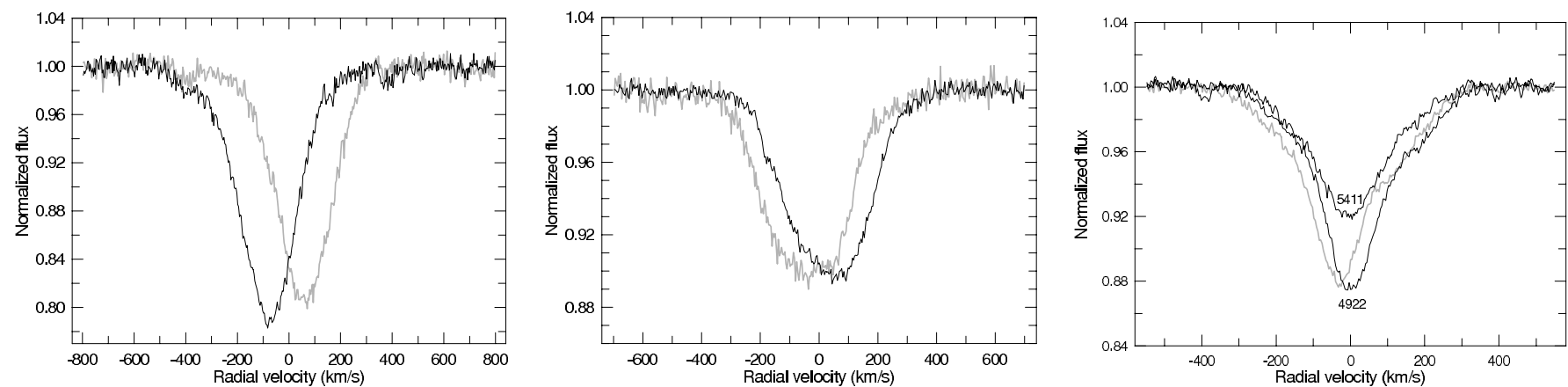

Fig. 6. Examples of profiles of helium lines of three stars. Left: HD 130298; He II line $5411 \AA$ at JD 2454211 (black) and 2454627 (gray). Middle: HD 163892 He I line $4922 \AA$ at JD 2454956 (black) and 2456097 (gray). Right: HD 167263; spectra of JD 2453483 (only He I $4922 \AA$ A) and 2454954 (He I 4922 and He II line $5411 \AA$ ).

Table 6. Elements of the orbits of five binaries with new periods.

\begin{tabular}{lccccc}
\hline \hline Element & HD 97166 & HD 115455 & HD 123590 & HD 130298 & HD 163892 \\
\hline Number of FEROS spectra & 12 & 15 & 26 & 11 & 11 \\
Period [days] & $57.733(17)$ & $15.08410(31)$ & $58.867(6)$ & $14.6302(6)$ & $7.834673(33)$ \\
$T_{\text {periastron RJD }}$ & $49140.4(1.5)$ & $40000.23(39)$ & $53894.75(25)$ & $53998.21(3)$ & $40003.74(6)$ \\
$e$ & $0.419(18)$ & $0.195(17)$ & $0.148(4)$ & $0.469(8)$ & $0.065(15)$ \\
$\omega[\mathrm{deg}]$ & $47(8)$ & $213(7)$ & $234.3(1.6)$ & $324.4(1.3)$ & $112(26)$ \\
$K_{1}\left[\mathrm{~km} \mathrm{~s}^{-1}\right]$ & $89.5(4.8)$ & $52.1(1.4)$ & $43.8(7)$ & $72.8(7)$ & $40.5(1.5)$ \\
$K_{2}\left[\mathrm{~km} \mathrm{~s}^{-1}\right]$ & $112.1(3.3)$ & $104.3(1.6)$ & $67.2(2)$ & & \\
$m_{2} / m_{1}$ & $0.799(20)$ & 0.500 & $0.653(16)$ & & \\
$m_{1} \sin ^{3} i\left[M_{\odot}\right]$ & 20.4 & 3.76 & 4.89 & & \\
$m_{2} \sin ^{3} i\left[M_{\odot}\right]$ & 16.3 & 1.88 & 3.19 & & \\
$V \gamma_{\text {pri }}\left[\mathrm{km} \mathrm{s}^{-1}\right]$ & $-2.6(3.4)$ & $-15.1(1.0)$ & $-7.4(0.8)$ & $-38.9(6)$ & $2.8(1.0)$ \\
$V \gamma_{\mathrm{sec}}\left[\mathrm{km} \mathrm{s}^{-1}\right]$ & $-9.1(4.3)$ & $-19.0(1.9)$ & $-7.1(0.7)$ & & \\
$a \sin i\left[R_{\odot}\right]$ & 212 & 49.0 & 80.5 & & 1.8 \\
$\mathrm{rms}\left[\mathrm{km} \mathrm{s}^{-1}\right]$ & $2.8(\mathrm{pri}) 3.5(\mathrm{sec})$ & $3.6(\mathrm{pri}) 5.4(\mathrm{sec})$ & $4.1(\mathrm{pri}) 0.7(\mathrm{sec})$ & $1.5(\mathrm{FEROS})$ & \\
\hline
\end{tabular}

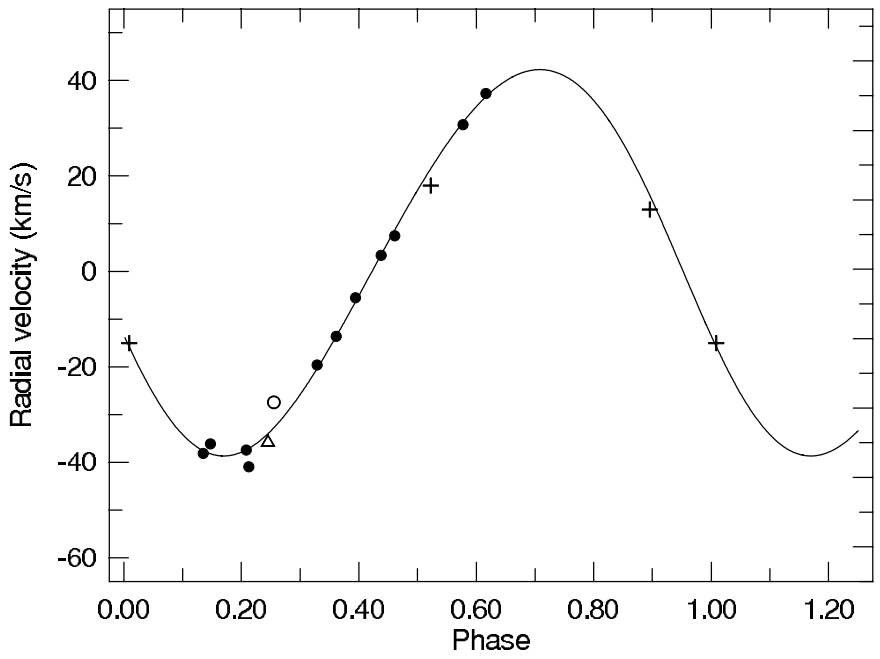

Fig. 7. Radial velocity curve of HD 163892. Full circles - FEROS, triangle - Conti et al. (1977), open circle - SL, crosses - Feast et al. (1963).

Although the secondary velocities could still only be measured at a few phases, a better determination of the secondary semiamplitude $K_{2}$ is now possible.

\subsubsection{Spectroscopy}

The FEROS spectra are listed in Table 7. Examples of the spectra taken near quadratures are shown in Fig. 8. In Table 7, the
Table 7. FEROS spectra of KX Vel.

\begin{tabular}{lrrrr}
\hline \hline $\begin{array}{l}\text { HJD } \\
-2400000\end{array}$ & $\begin{array}{r}\text { Exp. time } \\
\mathrm{s}\end{array}$ & Phase & $\begin{array}{r}\text { RV Pri } \\
\mathrm{km} \mathrm{s}^{-1}\end{array}$ & $\begin{array}{r}\text { RV Sec } \\
\mathrm{km} \mathrm{s}^{-1}\end{array}$ \\
\hline 53371.8581 & 90 & 0.5789 & 19.5 & 28.2 \\
53484.5784 & 150 & 0.8639 & 101.1 & -102.8 \\
53484.5808 & 150 & 0.8640 & 100.6 & -101.3 \\
55904.8770 & 120 & 0.8703 & 104.1 & -107.7 \\
55909.8645 & 120 & 0.0599 & -17.1 & 99.0 \\
\hline
\end{tabular}

average values of velocities obtained for the He I lines 4713, 4922, 5876, and $6678 \AA$ are listed. A simplex-based parameter optimization procedure was used to adjust the orbital elements by a simultaneous fit of the primary and secondary RVs. The MLD had also used two measurements of the secondary line He I $4713 \AA$ at phases 0.13 and 0.17 , but now we consider the line too weak and the RVs are certainly affected by the vicinity of the primary line. These two RVs were therefore not included in our RV sample for the parameter determination. All RVs measured on electronic spectra are plotted in Fig. 9. The orbital elements can be found in Table 8 .

The measured FWHM widths can be used to determine rotational velocities $V_{\text {rot }} \sin i$. For instance, Slettebak et al. (1975) has calibrated the FWHM versus $V_{\text {rot }} \sin i$ relation based on the spectra of a set of early-type standard stars, and Daflon et al. (2007) also derived such a correlation between the FWHM of helium lines in synthetic non-LTE spectra and the rotational 


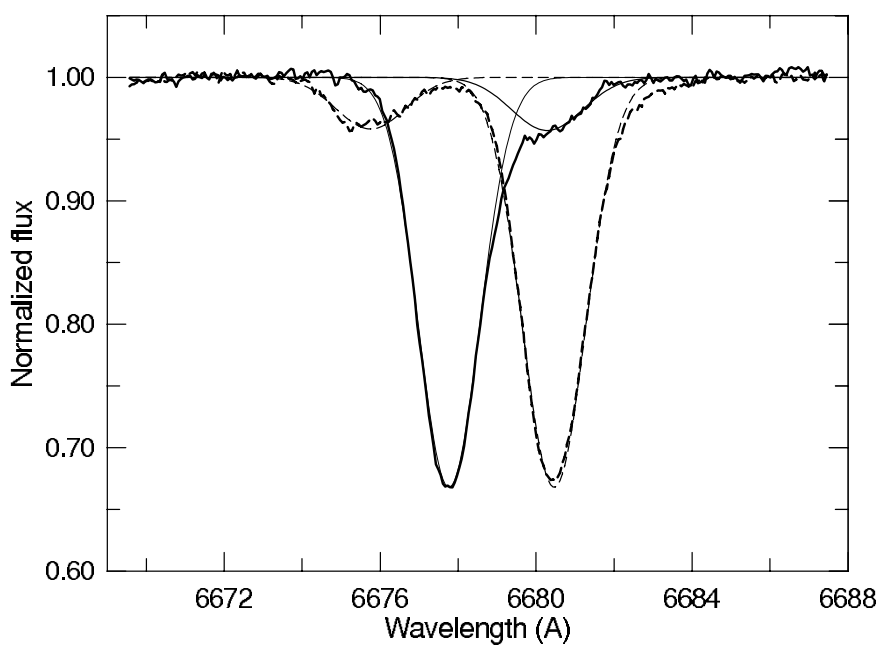

Fig. 8. The He I $6678 \AA$ line in two spectra of KX Vel taken near quadratures at JD 2455904 (dashed lines) and JD 2455909 (full lines).

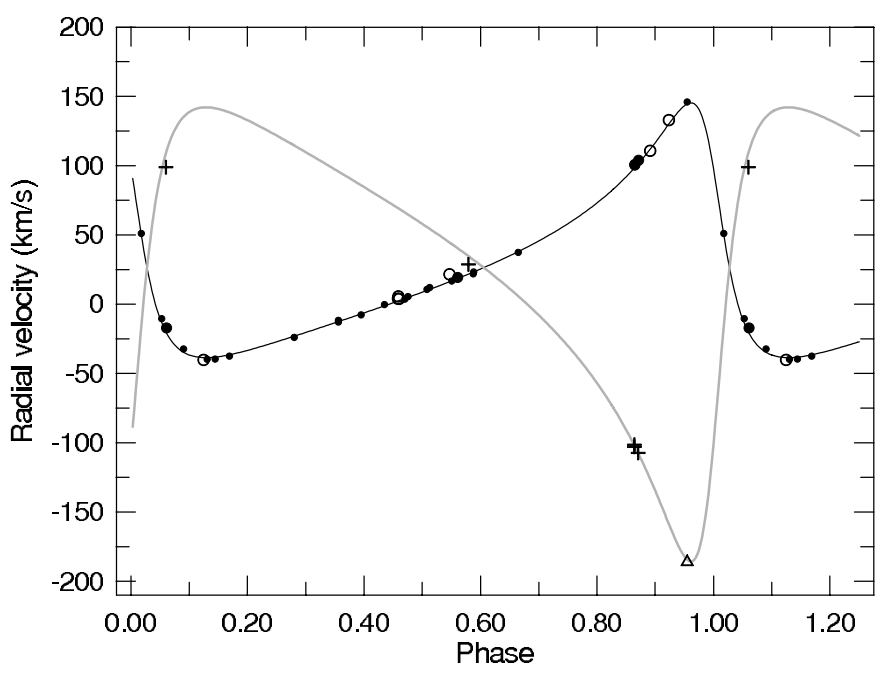

Fig. 9. Radial velocities of KX Vel. Primary RVs: full circles - FEROS, open circles - SL, small full circles - MLD. Secondary RVs: crosses FEROS, triangle - MLD.

Table 8. Elements of the orbit of KX Vel.

\begin{tabular}{lc}
\hline \hline Inclination [deg] & $74.6(7)$ \\
Period [days] & $26.30575(13)$ \\
$T_{\text {periastron }}$ & $49147.711(22)$ \\
$e$ & $0.598(6)$ \\
$\omega[\mathrm{deg}]$ & $60.3(4)$ \\
$K_{1}\left[\mathrm{~km} \mathrm{~s}^{-1}\right]$ & $92.8(9)$ \\
$K_{2}\left[\mathrm{~km} \mathrm{~s}^{-1}\right]$ & $163.5(2.7)$ \\
$m_{1} \sin ^{3} i\left[M_{\odot}\right]$ & $15.1(4)$ \\
$m_{2} \sin ^{3} i\left[M_{\odot}\right]$ & $8.6(2)$ \\
$V \gamma_{\text {pri }}\left[\mathrm{km} \mathrm{s}^{-1}\right]$ & $27.1(4)$ \\
$a \sin i\left[R_{\odot}\right]$ & $106.8(2.1)$ \\
\hline
\end{tabular}

velocities. We used a similar calibration by Munari \& Tomasella (1999) and the measured FWHM of the He I $5876 \AA$ line to derive the rotational velocity $V_{\text {rot }} \sin i$ as $44 \pm 4 \mathrm{~km} \mathrm{~s}^{-1}$ for both components of $\mathrm{KX}$ Vel. From Fig. 8, it is evident that He I $6678 \AA$ line profiles of both binary components can be well represented by Gaussian profiles.
The spectral classification, according to the Yerkes workers (Morgan et al. 1955; Hiltner et al. 1969), is B0 III, but others give O9.5 II, B0 Ib, or B1 Ib. However, according to Fraser et al. (2010), the star is not a supergiant. We therefore checked the classification: the $\log W^{\prime}$ criterion (Conti \& Alschuler 1971), although used in extrapolation, suggests that the type is B 0.5 . To decide about the luminosity class, the $u v b y$ photometry and $\mathrm{H} \beta$ index can help. The measured $\beta$ value is 2.588 (Hauck $\&$ Mermilliod 1998). According to Crawford (1978), $\beta$ should be 2.577 and 2.606 for the luminosity classes III and V, respectively. Therefore, the luminosity class of KX Vel might be closer to IV than III. Such a luminosity also agrees with the He I line $4143 \AA$.

\subsubsection{Comparison to synthetic spectra}

The available FEROS spectra of KX Vel are high in quality (with a signal-to-noise ratio exceeding 200) and, hence, allow for a quantitative spectral analysis with the aim to determine the atmospheric parameters of the binary components. We applied a new method developed by Irrgang et al. (2014) for an objective spectroscopic analysis of early-type single and binary stars. The approach is based on a grid of synthetic spectra calculated under the assumption of line-blanketed, plane-parallel model atmospheres with appropriate non-LTE modifications. Using the concept of $\chi^{2}$ minimization, the method is able to derive all essential atmospheric parameters, RVs, and element abundances. It is also applicable to composite spectra of double-lined spectroscopic binaries, if the spectra are obtained at orbital phases, where the spectral lines of both components are not severely blended. We, therefore, used the near-quadrature spectrum of KX Vel of JD 2455904 (phase 0.85; the dashed line in Fig. 8).

The composite spectrum is best reproduced by the following parameters: the primary component has an effective temperature of $T_{\mathrm{eff}, 1}=29710 \pm 40$ (stat.) \pm 600 (sys.) $\mathrm{K}$ and the surface gravity $\log g_{1}=3.669_{-0.004-0.1}^{+0.005+0.1}$; the secondary values are $T_{\text {eff, } 2}=$ $28070_{-170-870}^{+140+610} \mathrm{~K}$ and $\log g_{2}=4.343_{-0.021-0.434}^{+0.016+0.310}$. The RVs obtained from the cross-correlation of the complete observed spectrum with its best-matching synthetic representation are $V_{\text {rad, } 1}=$ $104.0_{-0.1-0.1}^{+0.2+0.1} \mathrm{~km} \mathrm{~s}^{-1}$ and $V_{\text {rad, } 2}=-110.9_{-1.0-0.4}^{+0.8+0.6} \mathrm{~km} \mathrm{~s}^{-1}$, which are in good agreement with the values +104.1 and $-107.7 \mathrm{~km} \mathrm{~s}^{-1}$ derived from our Gaussian fit of four He I lines (see Table 7). The ratio of the secondary to primary component effective surface areas $A_{\text {eff, }} / A_{\text {eff,p }}$, is $0.218_{-0.002-0.011}^{+0.003+0.014}$. This fit parameter weighs the flux contributions of both stars to the composite spectrum and assumes a constant surface brightness over the stellar disks. Hence, it is not identical to the actual geometric ratio of surface areas because, for example the effect of limb darkening is not included. However, by taking the ratio of effective surface areas, these shortcomings might cancel out, so that $A_{\text {eff,s }} / A_{\text {eff,p }}$ is supposed to be very close to the true geometric ratio.

Single-star evolutionary tracks by Ekström et al. (2012) are used to determine the stellar masses $\left(M_{1}=20.2_{-1.5 \text { (sys. })}^{+1.8 \text { (sys. }} M_{\odot}\right.$, $\left.M_{2}=11.5_{-0.5}^{+2.0} M_{\odot}\right)$, ages $\left(\tau_{1}=7_{-1}^{+1} \mathrm{Myr}, \tau_{2}=0_{-0}^{+10} \mathrm{Myr}\right)$, luminosities $\left(\log L_{1} / L_{\odot}=4.92 \pm 0.12, \log L_{2} / L_{\odot}=3.93_{-0.07}^{+0.42}\right)$, and radii $\left(r_{1}=10.9_{-1.6}^{+1.7} R_{\odot}, r_{2}=3.9_{-0.1}^{+2.9} R_{\odot}\right)$ from the position of the stars in the $\left(T_{\text {eff }}, \log g\right)$ diagram.

\subsubsection{Impact of the eclipse light curve}

It is a remarkable and an unfortunate fact that the photometric coverage of the eclipse is still limited to the observations by 
P. Mayer et al.: Several southern OB binaries

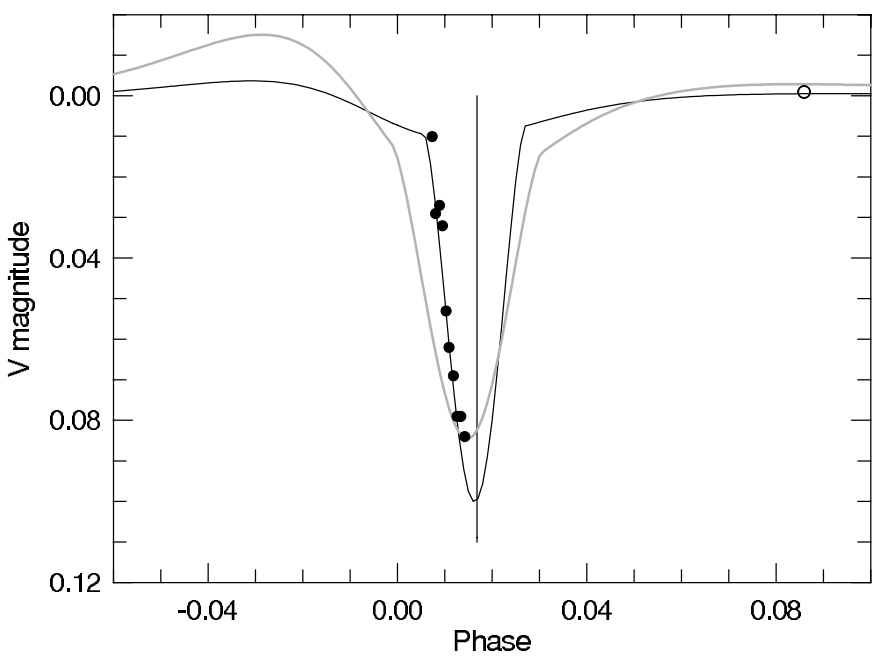

Fig. 10. KX Vel photometry by Balona \& Laing (1986). The open circle at the right represents the level of the measurements out of minimum. The black curve corresponds to the solution with $i=74.6^{\circ}$; the gray curve to the solution with $i=60^{\circ}$. The vertical line is drawn at the phase of the conjunction according to the FOTEL solution of the spectroscopy. The phase is calculated from the time of periastron.

Table 9. Parameters of the binary KX Vel.

\begin{tabular}{lcc}
\hline \hline Parameter & Primary & Secondary \\
\hline Mass $\left[M_{\odot}\right]$ & $16.8(5)$ & $9.5(3)$ \\
Radius $\left[R_{\odot}\right]$ & $12.6(5)$ & $5.9(3)$ \\
$\log g$ & $3.46(3)$ & $3.87(3)$ \\
\hline
\end{tabular}

Balona \& Laing (1986) for more than 20 years after the discovery of the eclipsing nature. Nevertheless, based on a more accurate ephemeris and better knowledge of the orbit, we tried to model the observed part of the eclipse using the code FOTEL (Hadrava 2004). We read the KX Vel magnitudes from Fig. 1 of the Balona \& Laing (1986) paper. The phase of the minimum is accurately known from spectroscopy; therefore, the incomplete light curve defines the shape of the minimum.

However, the partial coverage of the eclipse does not allow for an unbiased determination of the light curve parameters. It is namely the width of the eclipse, which is defined by the observations and which depends mostly on $r_{1}+r_{2}$ and inclination. So, we fitted the observations by adjusting the parameters $r_{1}+r_{2}$ and the inclination to achieve the best fit quality as judged by the $\chi^{2}$ sum. Hereby, we assumed a fixed ratio $r_{2} / r_{1}=0.467$ and luminosity ratio $L_{V, 2} / L_{V, 1}=0.2355$, which resulted from the spectroscopic analysis described in the previous section. With the mentioned constraints, we obtained $r_{1}+r_{2}=0.167 \pm 0.003$ (i.e. $r_{1}=0.114 \pm 0.004$ and $\left.r_{2}=0.053 \pm 0.002\right)$ and $i=74.6 \pm 0.7$. The parameter errors were assesed by confining the range of possible values of the $r_{2} / r_{1}$ ratio used for the light curve solution. The respective representation of the light curve is shown in Fig. 10, and the corresponding absolute masses and radii are listed in Table 9. The semimajor axis is 110.7 (2.2) $R_{\odot}$.

The primary mass comes out as $16.8 M_{\odot}$. It must be noted that the difference of the primary evolutionary and dynamical masses is considerable. There are two possibilities to end up with a larger dynamical mass - either the RV amplitude had to be larger, which can be ruled out by our RV curve (see Fig. 9), or the orbital inclination had to be as low as $\approx 60^{\circ}$. In this case no eclipses would occur, if the radii of our solution are assumed.

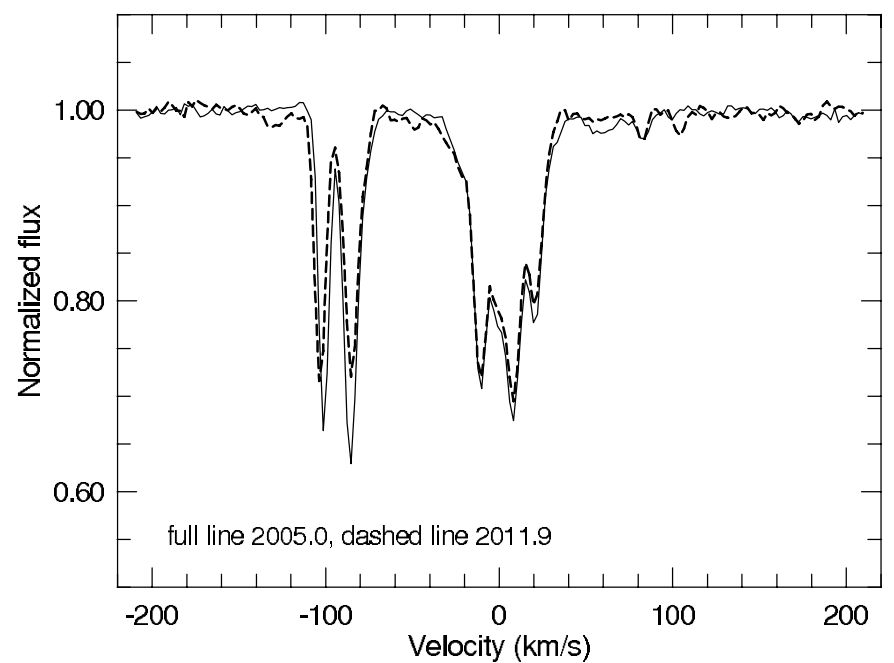

Fig. 11. The profile of the interstellar line Ca II 3933.66 in the spectrum of $\mathrm{KX}$ Vel; the velocity scale is relative to $V_{\mathrm{LSR}}$.

At such a low inclination, the observed eclipse depth could only be reproduced by radii, which are $56 \%$ larger than those following from our analysis. As illustrated in Fig. 10, the corresponding light curve (gray line) is far from being an acceptable representation of the observations. Hence, it must be concluded that the difference between the dynamical and evolutionary masses is real.

With the new radial velocities, the earlier published ephemeris changes only little. The primary and secondary minimum phases of this eccentric system should be 0.0170 and 0.7485 , respectively. However, for the given parameters, no secondary eclipse can be expected nor is it actually evident from the photometric observations.

\subsubsection{Interstellar lines}

The binary KX Vel is located in the field of the Vela OB 1 association, for which Humphreys (1978) gives a distance modulus of 11.37 , while KX Vel is only at about 10.0. Therefore, the star must be a foreground object. However, it is actually located behind the Vela supernova remnant and, therefore, displays a rich set of interstellar lines: at least five interstellar clouds can be recognized in the Ca II lines 3934 and $3968 \AA$, of which three are also apparent in the $\mathrm{Na}$ II lines. Interstellar $\mathrm{Ca}$ II and $\mathrm{Na}$ II lines in KX Vel spectra were studied by Danks \& Sembach (1995, see their Fig. 2) and by Cha \& Sembach (2000), with profiles shown in their Fig. 10. For these lines, Cha \& Sembach (2000) found changes of their profiles and velocities. With the new spectra, we can confirm changes of all interstellar features. In Fig. 11, two spectra with a time difference of seven years are superimposed (to be consistent with Cha \& Sembach, a correction of $-13.1 \mathrm{~km} \mathrm{~s}^{-1}$ to LSR was applied). Although the changes of the velocities are only minor (the largest one being observed for the component now at $-105 \mathrm{~km} \mathrm{~s}^{-1}$, while it was at $-98 \mathrm{~km} \mathrm{~s}^{-1}$ in the year 1993), changes of the profiles, namely, the decrease of the equivalent width of the component with RV $-88 \mathrm{~km} \mathrm{~s}^{-1}$, are considerable.

\section{2. $H D 167263$}

Radial velocities of this binary (16 Sgr, O9.5 II-III, $V=5.98$ ) were published by Feast et al. (1955), Garmany et al. (1980) 
Table 10. FEROS spectra and RVs of HD 167263.

\begin{tabular}{llrl}
\hline \hline HJD & $\begin{array}{l}\text { Exp. } \\
-2400000\end{array}$ & $\begin{array}{r}\text { Narrow line } \\
\text { time s }\end{array}$ & Phase \\
\hline 53482.9206 & 300 & -35.6 & 0.0620 \\
53482.9247 & 300 & -35.7 & 0.0623 \\
53855.9301 & 400 & -12.1 & 0.2919 \\
54953.9327 & 300 & -1.0 & 0.3339 \\
54953.9370 & 300 & -1.0 & 0.3342 \\
54976.8684 & 350 & -31.9 & 0.1311 \\
55699.9165 & 300 & -2.2 & 0.7909 \\
\hline
\end{tabular}

Table 11. Elements of the orbit of HD 167263.

\begin{tabular}{lc}
\hline \hline Period [days] & $12.76123(22)$ \\
\hline$T_{\text {periastron }}$ & $54005.3(7)$ \\
$e$ & $0.181(60)$ \\
$\omega[\mathrm{deg}]$ & $156(19)$ \\
$K_{1}\left[\mathrm{~km} \mathrm{~s}^{-1}\right]$ & $22.1(2.8)$ \\
$V \gamma_{\text {pri }}\left[\mathrm{km} \mathrm{s}^{-1}\right]$ & $-4.5(1.2)$ \\
$\mathrm{rms}\left[\mathrm{km} \mathrm{s}^{-1}\right]$ & 6.1 \\
\hline
\end{tabular}

and SL. SL found a period of 14.75825 , noting that a shorter period of 12 .74 would also be possible. This object is a known speckle binary with an estimated orbital period of 130 years (Mason et al. 1998). A preliminary value for the magnitude difference between the two components was 2 mag. However, a more recent value of this difference is only $0{ }^{\mathrm{m}} 4^{2}$. Three published interferometric measurements cover 17.6 years, and the position angle changed by $16: 3$, so any attempt to calculate the visual orbit appears as premature. It seems the orbital period is probably longer than 130 years.

If the latter small value of the magnitude difference is valid, the spectral lines of both speckle components should certainly be visible in the FEROS spectra. The line profiles consist of a narrow component, which represents the primary of the spectroscopic binary for which RVs had previously been derived, and a wide component, which would not be recognized in spectra of lower resolution. Radial velocities of the narrow parts can be well measured; RVs originating from various lines were found to differ by less than $\approx 3 \mathrm{~km} \mathrm{~s}^{-1}$. The wide part can be measured only approximately; differences might reach $\approx 10 \mathrm{~km} \mathrm{~s}^{-1}$, as seen in Fig. 6. Very likely, RVs of the wide profile parts are also changing but not in antiphase with the RVs of the narrow parts. Therefore, each of the interferometric components might be a binary itself.

It appears that the narrow profile originates in the fainter interferometric component. The spectra and measured RVs are listed in Table 10. The new RVs do not fit the longer period proposed by SL but are in better agreement with one of their suggested shorter periods. The corresponding orbital elements are listed in Table 11. The RV curve is rather noisy (Fig. 12), which might occur because the positions of the narrow line components were affected by the wider ones in spectra of lower dispersion. With the small number of spectra, their disentangling is uncertain; namely, finding any difference in spectral types of both components is difficult.

\footnotetext{
2 Fourth Catalog of Interferometric Measurements of Binary Stars, U.S. Naval Observatory, Washington, D.C.
}

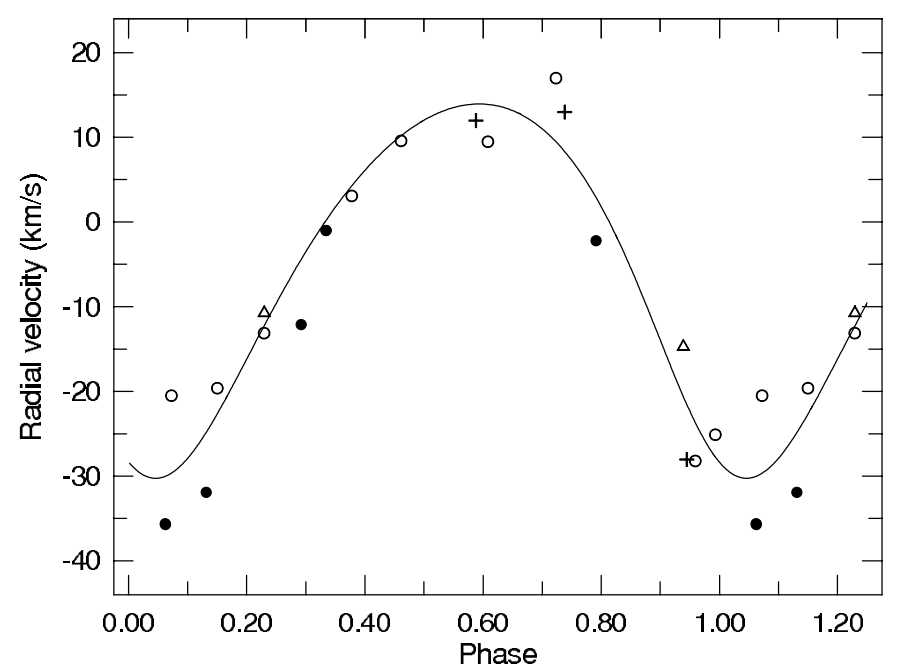

Fig. 12. Radial velocity curve of HD 167263; full circles - FEROS spectra, crosses - Feast et al. (1955), open circles - Garmany et al. (1980), triangles $-\mathrm{SL}$.

\section{Conclusions}

We present the results of a spectroscopic analysis of seven OBtype binaries. New and improved orbital and stellar parameters for these members of the important and rare class of early-type systems were obtained. It was demonstrated that a modest number of spectra were sufficient to find the period and other parameters of these binaries. With the exception of HD 123590 , the number of available RVs for our objects were relatively low. Additional spectra would be certainly welcome to confirm our values and to improve their accuracy. In some cases, it turns out that it is not possible to find lines of the secondary even with spectra of high signal-to-noise ratio. It can therefore be anticipated that cases with small mass ratios $M_{2} / M_{1}$ are probably not uncommon.

Using the spectroscopic analysis and the partial light curve of KX Vel, we were able to obtain acceptable values of masses and radii. These parameters, however, better agree with a less luminous spectral classification than what is used now. New data, photometry, and spectroscopy should be obtained to make these conclusions more reliable.

There are more than 100 binaries among southern OB objects; but the fundamental parameters for only less than half of them are known. Determining elements for more systems is necessary to improve the statistics of mass ratios and other parameters of OB-type binaries, and, of course, to give a better comparison of actually observed quantities to the predictions of the theories of stellar formation, structure, and evolution for this class of objects.

Acknowledgements. P.M. was supported by the grant P209/10/0715 of the Czech Science Foundation and also by the research program MSM0021620860. We kindly acknowledge helpful and valuable suggestions made by the referee Dr. Otmar Stahl.

\section{References}

Balona, L. A., \& Laing, J. D. 1986, MNRAS, 223, 621

Cha, A. N., \& Sembach, K. R. 2000, ApJS, 126, 399

Chini, R., Hoffmeister, V. H., Nasseri, A., Stahl, O., \& Zinnecker, H. 2012, MNRAS, 424, 1925

Conti, P. S., \& Alschuler, W. R. 1971, ApJ, 170, 325 
P. Mayer et al.: Several southern OB binaries

Conti, P. S., Leep, E. M., \& Lorre, J. J. 1977, ApJ, 214, 759

Crampton, D. 1972, MNRAS, 158, 85

Crawford, D. L. 1978, AJ, 83, 48

Daflon, S., Cunha, K., de Araújo, F. X., Wolff, S., \& Przybilla, N. 2007, AJ, 134, 1570

Danks, A. C., \& Sembach, K. R. 1995, AJ, 109, 2627

Ekström, S., Georgy, C., Eggenberger, P., et al. 2012, A\&A, 537, A146

Feast, M. W., Thackeray, A. D., \& Wesselink, A. J. 1955, MmRAS, 67, 51

Feast, M. W., Thackeray, A. D., \& Wesselink, A. J. 1963, MmRAS, 68, 1

Fraser, M., Dufton, P. L., Hunter, I., \& Ryans, R. S. I. 2010, MNRAS, 404, 1306

Garmany, C. D., Conti, P. S., \& Massey, P. 1980, ApJ, 242, 1063

Garrison, R. F., Hiltner, W. A., \& Schild, R. E. 1977, ApJS, 35, 111

Hadrava, P. 2004, Publications of the Astronomical Institute of the Czechoslovak Academy of Sciences, 92, 1

Hauck, B., \& Mermilliod, M. 1998, A\&AS, 129, 431

Humphreys, R. M. 1978, ApJS, 38, 309

Irrgang, A., Przybilla, N., Heber, U., et al. 2014, A\&A, 565, A63

Lanz, T., \& Hubeny, I. 2007, ApJS, 169, 83
Maíz-Apellániz, J., Walborn, N. R., Galué, H. Á., \& Wei, L. H. 2004, ApJS, 151, 103

Martins, F., Schaerer, D., \& Hillier, D. J. 2005, A\&A, 436, 1049

Mason, B. D., Gies, D. R., Hartkopf, W. I., et al. 1998, AJ, 115, 821

Mayer, P., Lorenz, R., \& Drechsel, H. 1997, A\&A, 320, 109

Mayer, P., Harmanec, P., \& Pavlovski, K. 2013, A\&A, 550, A2

Moffat, A. F. J., \& Vogt, N. 1975, A\&AS, 20, 85

Morgan, W. W., Code, A. D., \& Whitford, A. E. 1955, ApJS, 2, 41

Munari, U., \& Tomasella, L. 1999, A\&A, 343, 806

Slettebak, A., Collins, II, G. W., Parkinson, T. D., Boyce, P. B., \& White, N. M. 1975, ApJS, 29, 137

Sota, A., Maíz Apellániz, J., Walborn, N. R., et al. 2011, ApJS, 193, 24

Sota, A., Maíz Apellániz, J., Morrell, N. I., et al. 2014, ApJS, 211, 10

Stickland, D. J., \& Lloyd, C. 2001, The Observatory, 121, 1

Vázquez, R. A., Baume, G. L., Feinstein, C., Nuñez, J. A., \& Vergne, M. M. 2005, A\&A, 430, 471

Walborn, N. R. 1972, AJ, 77, 312

Walborn, N. R. 1973, AJ, 78, 1067 